\title{
Goldstone Theorem and Diquark Confinement Beyond Rainbow-Ladder Approximation
}

\author{
A. Bender, C. D. Roberts and L. v. Smekal \\ Physics Division, Bldg. 203, \\ Argonne National Laboratory, Argonne IL 60439-4843
}

\begin{abstract}
The quark Dyson-Schwinger equation and meson Bethe-Salpeter equation are studied in a truncation scheme that extends the rainbow-ladder approximation such that, in the chiral limit, the isovector, pseudoscalar meson remains massless. Quarkquark (diquark) correlations, which are bound in rainbow-ladder approximation, are destabilised by repulsive contributions that only appear at higher order in the Bethe-Salpeter kernel. The net effect of higher order terms on the meson boundstate masses is small.
\end{abstract}

Key words: Hadron spectroscopy; Confinement; Dyson-Schwinger equations; Bethe-Salpeter equation; Nonperturbative QCD phenomenology.

PACS Numbers: 12.38.Aw, 11.30.Rd, 24.85.+p, 12.38.Lg

\section{Introduction.}

The analytic structure of quark and gluon propagators can provide an understanding of confinement. Should these propagators have no Lehmann representation then a sufficient condition for confinement is satisfied; i.e., the absence of quark and gluon production thresholds in $\mathcal{S}$-matrix elements describing colour-singlet to singlet transitions. This in itself, however, neither precludes nor entails the existence of bound-states, whether coloured or not. The existence of two-body bound-states can only be established via the Bethe-Salpeter equation [BSE].

The homogeneous Bethe-Salpeter equation is derived under the assumption that the associated two-body $\mathcal{T}$-matrix has a pole in a given channel. The absence of a solution to the equation contradicts this assumption and establishes that no bound-state exists with the quantum numbers of the channel under consideration. 
The intrinsically nonperturbative nature of bound-state problems, and the complex structure of the QCD vacuum, suggests that one should employ dressed gluon and quark propagators in constructing the kernel of the BSE. These can be obtained via Dyson-Schwinger equations [DSEs]. The BetheSalpeter equation is one type of DSE.

There have been many studies of meson and diquark spectroscopy using this framework; a summary can be found in Ref. [1]. Typically, such studies employ an Ansatz for the gluon propagator in solving the rainbow-approximation quark-DSE and pair the input gluon propagator and calculated quark propagator to construct the dressed-ladder approximation kernel for the meson/diquark BSE. The BSE is then solved to obtain the spectrum. This rainbowladder truncation has the feature that Goldstone's theorem is manifest; i.e., in the chiral limit, when the current-quark mass $m_{q}=0$, the pion is a zeromass bound-state of a strongly-dressed quark and antiquark [2]. With a fewparameter model for the gluon propagator, it can be used to provide a good description of the light-light, light-heavy and heavy-heavy meson spectrum and decays [3]. However, it has the defect that it admits $(\overline{3})_{c}$-diquark boundstates [4]; such coloured states have not been observed.

Herein we consider a truncation scheme that allows for a systematic improvement in the construction of the kernels of the quark-DSE and meson/diquarkBSE. This procedure ensures that the pion remains a Goldstone boson at every order. The first correction to ladder-rainbow approximation introduces a repulsive term in the Bethe-Salpeter kernel. Using a model gluon propagator, singular in the infrared and with no Lehmann representation, we solve the quark DSE and obtain a quark propagator that also has no Lehmann representation. Pairing these gluon and quark propagators in the meson channel, the repulsive term is almost cancelled by attractive terms of the same order and therefore the higher order terms only lead to a small change in meson masses. However, due to the algebra of $S U(3)_{c}$, the repulsive term is stronger in the diquark channel and is not cancelled by the attractive terms. This entails that there is no stable diquark bound-state.

\section{Dyson-Schwinger and Bethe-Salpeter equations.}

In Euclidean metric, with $\gamma_{\mu}$ hermitian and $\left\{\gamma_{\mu}, \gamma_{\nu}\right\}=2 \delta_{\mu \nu}$, the propagator for a dressed quark has the form $S(p)=-i \gamma \cdot p \sigma_{V}\left(p^{2}\right)+\sigma_{S}\left(p^{2}\right)$. It is obtained as a solution of the quark DSE

$$
\begin{aligned}
& S^{-1}(p) \equiv i \gamma \cdot p+m_{q}+\Sigma(p) \\
& =i \gamma \cdot p+m_{q}+g^{2} \int \frac{d^{4} k}{(2 \pi)^{4}} \gamma_{\mu} \frac{\lambda^{a}}{2} S(k) \Gamma_{\nu}^{g}(k, p) \frac{\lambda^{a}}{2} D_{\mu \nu}(p-k),
\end{aligned}
$$

where $m_{q}$ is the current-quark mass, $\Gamma_{\mu}^{g}(k, p)$ is the dressed quark-gluon vertex and $D_{\mu \nu}(k)$ is the dressed gluon propagator, which, in Landau gauge, can be 
written

$$
g^{2} D_{\mu \nu}(k) \equiv\left(\delta_{\mu \nu}-\frac{k_{\mu} k_{\nu}}{k^{2}}\right) \Delta\left(k^{2}\right)
$$

The mass and Bethe-Salpeter amplitude for a quark-antiquark bound-state, $\Gamma_{M}$, is obtained by solving

$$
\begin{aligned}
& \Gamma_{M}^{E F}(p ; P)= \\
& \int \frac{d^{4} k}{(2 \pi)^{4}} K_{M}^{E F ; G H}(k, p ; P)\left(S\left(k+\frac{1}{2} P\right) \Gamma_{M}(k ; P) S\left(k-\frac{1}{2} P\right)\right)^{G H},
\end{aligned}
$$

where $K_{M}(k, p ; P)$ is the kernel, $P$ is the total-momentum of the system, $k, p$ are the internal and external relative quark-antiquark momenta and the superscripts are associated with the colour, flavour and Dirac structure of the amplitude; i.e., $E=\left\{i_{c}, i_{f}, i_{D}\right\}$.

The analogue of Eq. (3) for quark-quark systems is

$$
\begin{aligned}
& \Gamma_{D}^{E F}(p ; P)= \\
& \int \frac{d^{4} k}{(2 \pi)^{4}} K_{D}^{E F ; G H}(k, p ; P)\left(S\left(k+\frac{1}{2} P\right) \Gamma_{D}(k ; P) S^{T}\left(-k+\frac{1}{2} P\right)\right)^{G H},
\end{aligned}
$$

where " $T$ " denotes matrix-transpose. The absence of a solution to this equation entails that diquarks do not appear in the strong-interaction spectrum.

In the isovector channel, each contribution to $K_{M}^{E F ; G H}(k, p ; P)$ has a direct analogue in $K_{D}^{E F ; G H}(k, p ; P)$; an analogue that can be obtained via the replacement

$$
S(k) \gamma_{\mu} \frac{\lambda^{a}}{2} \rightarrow\left[\gamma_{\mu} \frac{\lambda^{a}}{2} S(-k)\right]^{T}
$$

$\left(\left\{\lambda^{a} / 2\right\}_{a=1}^{8}\right.$ are the generators of $\left.S U(3)_{c}\right)$ in each antiquark segment of the meson kernel, which can be traced unambiguously from the external antiquarkline of the meson amplitude. This means that there are no contributions in the diquark channel that are not also present in the isovector meson channel.

Rainbow-ladder approximation.

The rainbow approximation to the quark DSE is defined by the choice $\Gamma_{\mu}^{g}(k, p) \equiv$ $\gamma_{\mu}$ in Eq. (1); the ladder-approximation to the meson BSE by

$$
K_{M}^{E F ; G H}(k, p ; P)\left(S\left(k+\frac{1}{2} P\right) \Gamma_{M}(k ; P) S\left(k-\frac{1}{2} P\right)\right)^{G H}
$$




$$
\equiv-g^{2} D_{\mu \nu}(p-k)\left(\gamma_{\mu} \frac{\lambda^{a}}{2} S\left(k+\frac{1}{2} P\right) \Gamma_{M}(k ; P) S\left(k-\frac{1}{2} P\right) \gamma_{\nu} \frac{\lambda^{a}}{2}\right)^{E F}
$$

in Eq. (3).

In the chiral limit the rainbow-approximation DSE and the pseudoscalarmeson BSE in ladder-approximation are equivalent when $P^{2}=0$; i.e., one necessarily has a massless, pseudoscalar bound-state when chiral symmetry is dynamically broken[2]. Goldstone's theorem is manifest. In any truncation, such an identity between the quark DSE and the BSE in the isovector, pseudoscalar, meson channel is sufficient to ensure that this meson is a Goldstone boson [5]. This provides for a straightforward understanding of the dichotomy of the pion as both a Goldstone boson and quark-antiquark bound-state.

A gluon propagator that has no Lehmann representation may be interpreted as describing a confined particle. Such a gluon propagator can, via Eq. (1), yield a quark propagator with the same property [6]. Hence, in rainbow-ladder approximation, one can develop a spectroscopic model of mesons with confined quarks and gluons.

However, at this order the diquark BSE, Eq. (4), admits solutions; i.e., one has $(\overline{3})_{c}$ diquark states in the spectrum [4], which are not observed. (It follows from the algebra of the generators of $S U(3)_{c}$ that, in this approximation, there are no $(6)_{c}$ diquark solutions just as there are no $(8)_{c}$ meson solutions.)

\section{Next-order Truncation}

As the next level of truncation for the quark DSE we consider Eq. (1) with

$$
\Gamma_{\nu}^{g}(k, p)=\gamma_{\nu}+\frac{1}{6} \int \frac{d^{4} l}{(2 \pi)^{4}} g^{2} D_{\rho \sigma}(p-l) \gamma_{\rho} S(l+k-p) \gamma_{\nu} S(l) \gamma_{\sigma}
$$

This is the first-order correction of the vertex by the dressed-gluon propagator and the dressed-quark propagator, which is obtained as the solution of this DSE. Here we omit the explicit 3-gluon vertex that could contribute at this order. This entails that we explore 3 - and 4-body forces only to the extent that they are incorporated via the nonperturbative dressing of the gluon propagator.

For the purpose of illustration and clarity we employ a model gluon propagator [6] that allows for an algebraic solution of the Dyson-Schwinger and Bethe-Salpeter equations:

$$
\Delta\left(k^{2}\right) \equiv 16 \pi^{4} G \delta^{4}(k)
$$

in Eq. (2), where $G=\eta^{2} / 4$ with $\eta$ a mass scale. This one-parameter model 
possesses the infrared enhancement, due to the 3-gluon vertex, suggested by the studies of Refs. [7] but underestimates the interaction strength for $k^{2}>0$. This is appropriate in a study whose focus is confinement and dynamical chiral symmetry breaking, which are infrared effects. The qualitative features of our results are not sensitive to this choice.

Using Eqs. (7) and (8) in Eq. (1) one obtains】

$$
S^{-1}(p)=i \gamma \cdot p+m_{q}+G \gamma_{\mu} S(p) \gamma_{\mu}+\frac{1}{8} G^{2} \gamma_{\mu} S(p) \gamma_{\nu} S(p) \gamma_{\mu} S(p) \gamma_{\nu}
$$

Neglecting the $\mathrm{O}\left(G^{2}\right)$ term yields the rainbow-approximation quark DSE.

\section{Goldstone Theorem.}

An important consideration in extending the kernel of the Bethe-Salpeter equation is to ensure that in doing so one preserves the Goldstone boson character of the pion. To this end we observe that the ladder-approximation kernel in the meson BSE can be obtained directly from the expression for $\Sigma(p)$ in the rainbow-approximation quark DSE via the replacement

$$
\gamma_{\mu} S(k) \gamma_{\nu} \rightarrow \gamma_{\mu} S(k+P / 2) \Gamma_{M}(k, P) S(k-P / 2) \gamma_{\nu}
$$

which is illustrated in the top diagram of Fig. 1.

In the isovector channel, this procedure can be implemented at every order; i.e., in every term of the quark DSE one may sequentially replace each explicit, internal quark propagator in this way, which is illustrated for Eq. (9) in Fig. 1. This generates all contributions of a given order to the kernel and ensures that Goldstone's theorem is preserved at that order, as will become clear below. Applying the procedure to vacuum polarisation insertions does not generate additional terms in the isovector kernel; a fact that very much simplifies the study of isovector systems, allowing one to employ a model gluon propagator and maintain Goldstone's theorem.

These arguments are independent of the form of the model gluon propagator. The inclusion of 3- and 4-gluon vertices is a straightforward extension, which does not modify the conclusions.

The isoscalar channel receives additional contributions from vacuum polarisation diagrams. This may provide the basis for understanding the $\eta-\eta$ ' mass splitting in the present framework[8].

1 In deriving this equation we have used the identity

$\int d^{4} l \delta^{4}(l) \frac{p \cdot l k \cdot l}{l^{2}} f(k, p, l) \equiv \frac{1}{4} \int d^{4} l \delta^{4}(l) p \cdot k f(k, p, l)$ 

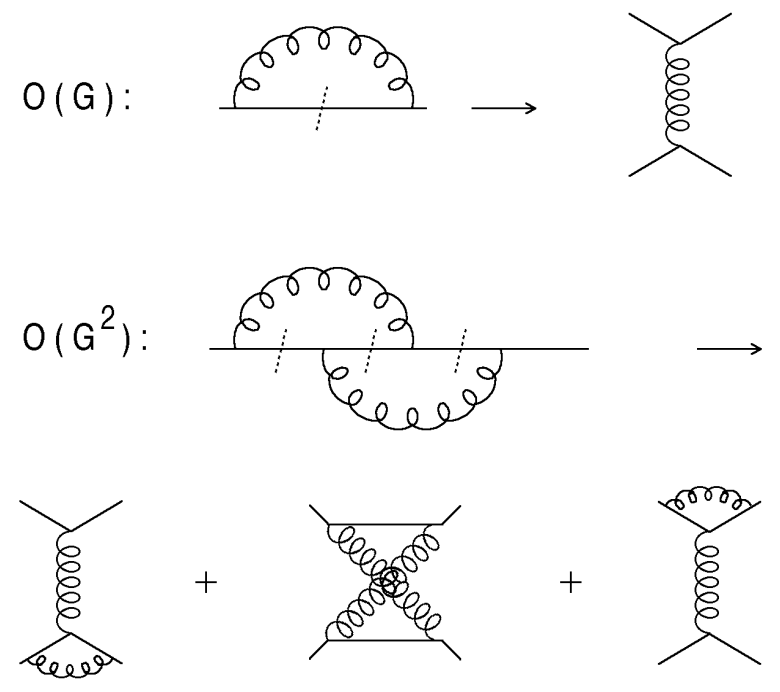

Fig. 1. The replacement procedure of Eq. (10) illustrated to $\mathrm{O}\left(G^{2}\right)$, which provides the kernel of the meson and diquark Bethe-Salpeter equations. The internal solid lines represent dressed quark and gluon propagators.

The full $\mathrm{O}\left(G^{2}\right)$ kernel for the isovector-meson Bethe-Salpeter equation is illustrated in Fig. 1. Using Eq. (8), the Bethe-Salpeter equation for $(1)_{c}$, isovector mesons is

$$
\begin{aligned}
& \Gamma_{M}(p ; P)=-G \gamma_{\mu} \chi_{M} \gamma_{\mu} \\
& -\frac{1}{8} G^{2} \gamma_{\mu}\left(S_{+} \gamma_{\nu} S_{+} \gamma_{\mu} \chi_{M}+\underline{S_{+} \gamma_{\nu} \chi_{M} \gamma_{\mu} S_{-}}+\chi_{M} \gamma_{\nu} S_{-} \gamma_{\mu} S_{-}\right) \gamma_{\nu}
\end{aligned}
$$

where $S_{ \pm} \equiv S(p \pm P / 2)$ and $\chi_{M} \equiv S_{+} \Gamma_{M}(p ; P) S_{-}$. Using Eq. (5) the BetheSalpeter equation for $(\overline{3})_{c}$, isovector diquarks can be written

$$
\begin{aligned}
& \Gamma_{D^{\overline{3}}}^{C}(p ; P)=-\frac{1}{2} G \chi_{D^{\overline{3}}}^{C} \gamma_{\mu}-\frac{1}{16} G^{2} \gamma_{\mu}\left(S_{+} \gamma_{\nu} S_{+} \gamma_{\mu} \chi_{D^{\overline{3}}}^{C}\right. \\
& \left.+5 \underline{S_{+} \gamma_{\nu} \chi_{D^{\overline{3}}}^{C} \gamma_{\mu} S_{-}}+\chi_{D^{\overline{3}}}^{C} \gamma_{\nu} S_{-} \gamma_{\mu} S_{-}\right) \gamma_{\nu},
\end{aligned}
$$

with $\Gamma_{D^{\overline{3}}} \equiv \Gamma_{D^{\overline{3}}}^{C} C$ and $\chi_{D^{\overline{3}}}^{C} \equiv S_{+} \Gamma_{D^{\overline{3}}}^{C}(p ; P) S_{-}$, where $C=\gamma_{2} \gamma_{4}$ is the charge conjugation matrix. In these equations the underlined term is the "crossedbox" contribution of Fig. 1.

Diquark bound-states.

At $\mathrm{O}(G)$ the only difference between Eqs. (11) and (12) is a $50 \%$ reduction in the coupling strength; i.e., the coupling is twice as strong in the meson equation. This follows from the algebra of the $S U(3)_{c}$ generators and entails [9] the existence of scalar and pseudovector diquark bound-states with $m_{q q}^{0^{+}}>$ $m_{\bar{q} q}^{0^{-}}$and $m_{q q}^{1^{+}}>m_{\bar{q} q}^{1^{-}}$. 
Of the terms in Eqs. (11) and (12) only the underlined one in each is repulsive in the $0_{\bar{q} q}^{-}, 1_{\bar{q} q}^{-}, 0_{q q}^{+}, 1_{q q}^{+}$channels. The numerical factors arise from the algebra of the $S U(3)_{c}$ generators and do not depend on the form of the gluon propagator. Relative to its companion, parenthesised contributions, this term is 5 -times as large in the diquark equation, which provides for the possibility that it eliminates diquark bound-states.

\section{Results and Conclusions}

In order to study the bound-state spectrum one must solve Eq. (9). Our results are illustrated in Fig. 2. The $\mathrm{O}\left(G^{2}\right)$ corrections become noticeable for $p^{2} \lesssim$ $\eta^{2} / 2$, which is important because it is the domain sampled in the BetheSalpeter equation. It is also important to note that one has dynamical chiral symmetry breaking for any $G>0$; i.e., for $m_{q}=0: \sigma_{S}(0)>0, \forall G>0$. Further, $\sigma_{V}$ and $\sigma_{S}$ have no pole on the real- $p^{2}$ axis. Therefore the quark propagator has no Lehmann representation and can be interpreted as describing a confined particle.

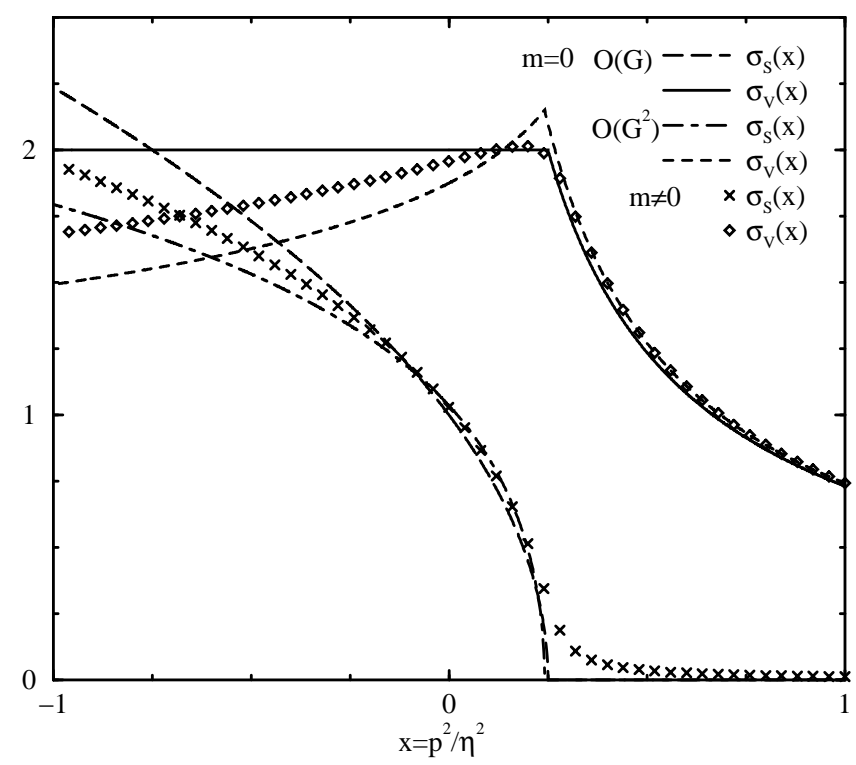

Fig. 2. We plot $\bar{\sigma}_{V}(x)$ and $\bar{\sigma}_{S}(x)$ obtained as the solution of Eq. (9); $\sigma_{V}\left(p^{2}\right)=\bar{\sigma}_{V}(x) / \eta^{2}, \sigma_{S}\left(p^{2}\right)=\bar{\sigma}_{S}(x) / \eta$.

The model gluon propagator of Eq. (8) entails that the bound-state constituents have zero relative momentum; i.e., $p=0$. In this case the most general form of the $0_{\bar{q} q}^{-}$Bethe-Salpeter amplitude is

$$
\Gamma_{M}^{0^{-}}(P)=\left[\theta_{1}^{0^{-}}\left(P^{2}\right)+i \frac{\gamma \cdot P}{\eta} \theta_{2}^{0^{-}}\left(P^{2}\right)\right] \gamma_{5}
$$


Substituting Eq. (13) into Eq. (11), with $p=0$, one obtains a $2 \times 2$ matrix eigen-value problem of the form $H \Theta=\Theta$; where the elements of $H$ depend on $P^{2}$, and $\Theta^{T}=\left(\theta_{1}, \theta_{2}\right)$. The eigen-value problem is solved when that $P^{2}$ is found for which $\operatorname{det}\left\{H\left(P^{2}\right)-I\right\}=0$; the eigen-vector follows immediately. The same procedure is followed in each of the other channels. The vectormeson Bethe-Salpeter amplitude has the form

$$
\Gamma_{M}^{1^{-}}(P)=\epsilon^{\lambda} \cdot \gamma \theta_{1}^{1^{-}}\left(P^{2}\right)+\frac{i}{\eta} \sigma_{\mu \nu} \epsilon_{\mu}^{\lambda} P_{\nu} \theta_{2}^{1^{-}}\left(P^{2}\right)
$$

with $\epsilon_{\mu}^{\lambda}(P), \lambda=0, \pm 1$, the polarisation vector: $\epsilon^{\lambda} \cdot P=0 ; \Gamma_{D^{\overline{3}}}^{0^{+}}$has the same form as the pseudoscalar meson amplitude in Eq. (13); and $\Gamma_{D^{\overline{3}}}^{1^{+} C}$ the same form as the vector-meson amplitude in Eq. (14).

Our chiral limit results are presented in Table 1. The $\mathrm{O}(G): \mathrm{O}(G)$ eigen-vectors are $\left(\theta_{1}^{0^{-}}, \theta_{2}^{0^{-}}\right)=(0.83,0.55),\left(\theta_{1}^{1^{-}}, \theta_{2}^{1^{-}}\right)=(1,0)$. At $\mathrm{O}\left(G^{2}\right): \mathrm{O}\left(G^{2}\right)$ they are $\left(\theta_{1}^{0^{-}}, \theta_{2}^{0^{-}}\right)=(0.87,0.49),\left(\theta_{1}^{1^{-}}, \theta_{2}^{1^{-}}\right)=(0.99,-0.12)$.

\section{Table 1}

Calculated meson and diquark masses, in $\mathrm{GeV}(\eta=1.06 \mathrm{GeV})$. The labels " $\mathrm{O}\left(G^{n}\right)$ : $\mathrm{O}\left(G^{m}\right)$ " mean that the solution of the $\mathrm{O}\left(G^{n}\right)$ quark Dyson-Schwinger equation was used in the $\mathrm{O}\left(G^{m}\right)$ Bethe-Salpeter equation. The non-zero quark mass was chosen to reproduce the experimental ratio $m_{\pi} / m_{\rho}$ at $\mathrm{O}\left(G^{2}\right): \mathrm{O}\left(G^{2}\right)$. "Unbound" means that there is no solution of the associated homogeneous Bethe-Salpeter equation for real $P^{2}$.

\begin{tabular}{llll}
$m_{q}=0$ & $\mathrm{O}(G): \mathrm{O}(G)$ & $\mathrm{O}(G): \mathrm{O}\left(G^{2}\right)$ & $\mathrm{O}\left(G^{2}\right): \mathrm{O}\left(G^{2}\right)$ \\
\hline$m_{\bar{q} q}^{0^{-}}$ & 0 & 0.30 & 0 \\
$m_{q q}^{0^{+}}$ & 1.19 & Unbound & Unbound \\
$m_{\bar{q} q}^{1^{-}}$ & 0.750 & 0.745 & 0.823 \\
$m_{q q}^{1^{+}}$ & 1.30 & Unbound & Unbound \\
\hline$m_{q}=0.012$ & & & \\
\hline$m_{\bar{q} q}^{0^{-}}$ & 0.140 & 0.328 & 0.136 \\
$m_{q q}^{0^{+}}$ & 1.21 & Unbound & Unbound \\
$m_{\bar{q} q}^{1^{-}}$ & 0.767 & 0.760 & 0.770 \\
$m_{q q}^{1^{+}}$ & 1.32 & Unbound & Unbound \\
\hline
\end{tabular}

The Goldstone theorem is manifest when the quark DSE and pseudoscalar meson BSE are truncated consistently; i.e, at $\mathrm{O}(G): \mathrm{O}(G)$ and $\mathrm{O}\left(G^{2}\right): \mathrm{O}\left(G^{2}\right)$. This can be shown analytically. If the dressing is inconsistent; e.g., $\mathrm{O}(G)$ : $\mathrm{O}\left(G^{2}\right)$, the pseudoscalar is half as massive as the vector meson.

The $\mathrm{O}\left(G^{2}\right)$ corrections only provide for a small (10\%) mass increase in the 
vector-meson channel, as one would expect of a weak, net-repulsive interaction. It is weak because of the cancellation between the vertex-correction and crossed-box contributions, which is a necessary consequence of the preservation of Goldstone's theorem.

In the diquark channel, however, where the coefficient of the repulsive term is larger and the cancellation incomplete, the $\mathrm{O}\left(G^{2}\right)$ corrections have the significant effect of eliminating the bound-state pole on the real- $P^{2}$ axis.

We note that the pseudovector, $\theta_{2}^{0^{-}}$, component of the pseudoscalar meson is a significant part of its Bethe-Salpeter amplitude whereas the tensor, $\theta_{2}^{1^{-}}$, component of the vector meson is small. This feature is also observed in the separable Ansatz studies of Ref. [10].

Our results for $m_{q} \neq 0$ are presented in Table 1. The $\mathrm{O}\left(G^{2}\right): \mathrm{O}\left(G^{2}\right)$ meson eigen-vectors are: $\left(\theta_{1}^{0^{-}}, \theta_{2}^{0^{-}}\right)=(0.86,0.51)$ and $\left(\theta_{1}^{1^{-}}, \theta_{2}^{1^{-}}\right)=(0.99,-0.13)$. The Goldstone boson character of the pseudoscalar is clear; i.e., at a consistent level of truncation its mass increases rapidly from zero as $m_{q}$ is increased. In contrast, the vector mass shifts upward by $<1 \%$. (The upward shift is $\approx 10 \%$ for $m_{q}=0$ because the difference between the $\mathrm{O}(G)$ and $\mathrm{O}\left(G^{2}\right)$ solutions of the quark DSE is greater in this case.) A non-zero current-quark mass introduces little quantitative and no qualitative change in the diquark channels; i.e., they remain unbound.

For $m_{q}=0.012 \mathrm{GeV}$ we have plotted $\operatorname{det}\left\{H\left(P^{2}\right)-I\right\}$ in Fig. 3. It illustrates the effect of the $\mathrm{O}\left(G^{2}\right)$ repulsive term in the Bethe-Salpeter kernel, which shifts the zero in the meson channel very little but completely eliminates it in the diquark channel. The additional repulsive strength in the diquark channel is amplified by the growth in $\sigma_{S}(x)$ for $x<0$; i.e., the feature of confinement manifest in the quark propagator, and due to the infrared enhancement of the gluon propagator, plays a role in destabilising the diquarks.

A motivation for our study is the observation that while the absence of a Lehmann representation for gluon and quark propagators ensures there are no gluons and quarks in the strong interaction spectrum, it neither entails nor precludes the existence of bound-states, whether coloured or not. We have shown that the rainbow-ladder truncation is peculiar in the sense that it alone has no repulsive terms in the BSE kernel. In every truncation beyond this there are both attractive and repulsive terms. We saw that in a consistent truncation there was almost complete cancellation between the $\mathrm{O}\left(G^{2}\right)$ attractive and repulsive terms in the meson channel. This is an indication of why the studies of meson spectroscopy and decays using a model gluon propagator in rainbow-ladder approximation have been successful. In the diquark channel the situation is quite different. The algebra of $S U(3)_{c}$ ensures that the coefficient of the $\mathrm{O}\left(G^{2}\right)$ repulsive term is larger and this, coupled with confinement 


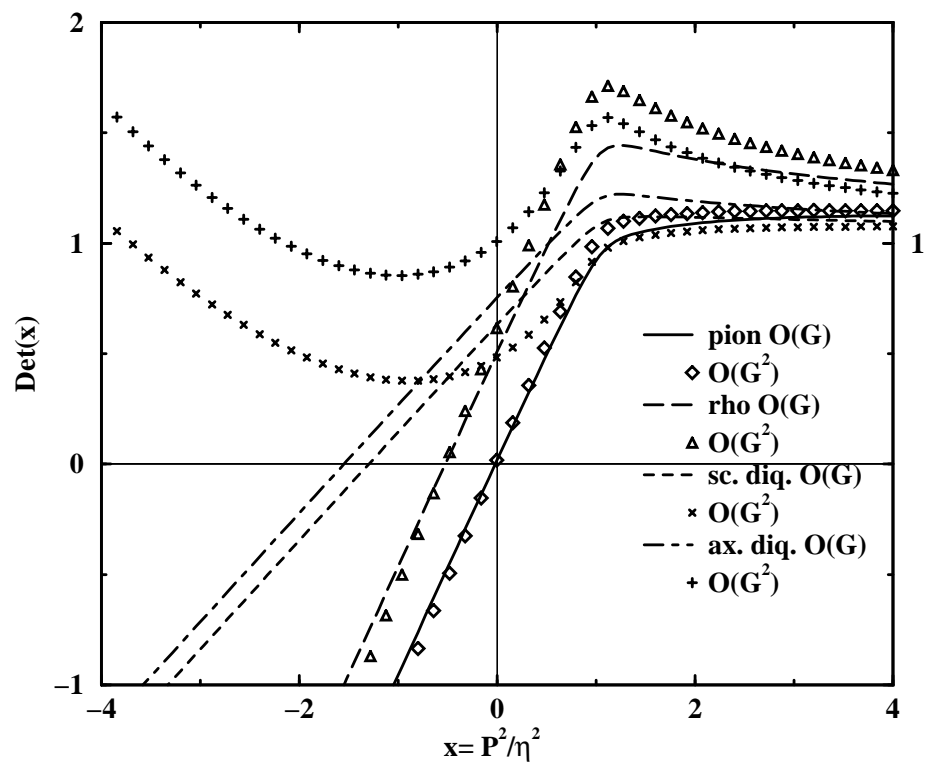

Fig. 3. $\operatorname{det}\left\{H\left(P^{2}\right)-I\right\}$ plotted as a function of $P^{2}$. This function vanishes at the square of the bound-state mass in the channel under consideration.

as manifest in the form of the quark propagator, entails that the repulsive effect survives to ensure the absence of a stable diquark bound-state. This effect cannot reasonably be reproduced in rainbow-ladder approximation. It is plausible that these features persist at higher order and with the inclusion of explicit $3-$ and 4 -gluon vertices.

\section{Acknowledgments.}

This work was supported by the US Department of Energy, Nuclear Physics Division, under contract number W-31-109-ENG-38. The calculations described herein were carried out using the resources of the National Energy Research Supercomputer Center.

\section{References}

[1] C. D. Roberts and A. G. Williams, Prog. Part. Nucl. Phys. 33 (1994) 477.

[2] R. Delbourgo and M. D. Scadron, J. Phys. G 5 (1979) 1631.

[3] P. Jain and H. J. Munczek, Phys. Rev. D 48 (1993) 5403; and references therein.

[4] J. Praschifka, R. T. Cahill and C. D. Roberts, Intern. J. Mod. Phys. A 4 (1989) 4929.

[5] H. J. Munczek, Phys. Rev. D 52 (1995) 4736. 
[6] H. J. Munczek and A. M. Nemirovsky, Phys. Rev. D 28 (1983) 181.

[7] M. Baker, J. S. Ball and F. Zachariasen, Nucl. Phys. B 186 (1981) 531; ibid 560; D. Atkinson, P. W. Johnson, W. J. Schoenmaker and H. A. Slim, Nuovo Cimento A 77, Series 11 (1983) 197; N. Brown and M. R. Pennington, Phys. Rev. D 39 (1989) 2723; A. Hauck, L. v. Smekal and R. Alkofer, in preparation.

[8] J. Kogut and L. Susskind, Phys. Rev. D 10 (1974) 3468; Y. Kurumashi, et al, Phys. Rev. Lett. 72 (1994) 3448; R. Alkofer, Hadronen in NambuJona-Lasinio-Modellen, Habilitationsschrift, Universität Tübingen (1995), and references therein.

[9] R. T. Cahill, C. D. Roberts and J. Praschifka, Phys. Rev. D 36 (1989) 2804.

[10] C. J. Burden, et al., Separable approximation to the Bethe-Salpeter equation in $Q C D$, to appear in the proceedings of "Lattice 95", Melbourne, Australia, June, 1995; C. J. Burden, Lu Qian, C. D. Roberts, P. C. Tandy and M. J. Thomson, Spectroscopy of light quark mesons (in preparation). 